\title{
New pattern of EGFR amplification in glioblastoma and the relationship of gene copy number with gene expression profile
}

\author{
Concha Lopez-Gines ${ }^{1}$, Rosario Gil-Benso ${ }^{1}$, Ruben Ferrer-Luna ${ }^{2}$, Rafael Benito ${ }^{1}$, Eva Serna ${ }^{3}$, \\ Jose Gonzalez-Darder ${ }^{4}$, Vicente Quilis ${ }^{4}$, Daniel Monleon ${ }^{5}$, Bernardo Celda ${ }^{2}$ and \\ Miguel Cerdá-Nicolas ${ }^{1}$ \\ ${ }^{1}$ Department of Pathology, University of Valencia, Valencia, Spain; ${ }^{2}$ Department of Quimica-Fisica, \\ University of Valencia, Valencia, Spain; ${ }^{3}$ UCIM, University of Valencia, Valencia, Spain; ${ }^{4}$ Department of \\ Neurosurgery, Hospital Clínico Universitario, Valencia, Spain and ${ }^{5}$ Fundación HCU, Labim, Valencia, Spain
}

\begin{abstract}
Gene amplification is a process that is characterized by an increase in the copy number of a restricted region in a chromosome arm, and is frequently associated with an overexpression of the corresponding amplified gene. Amplified DNA can be organized either as extrachromosomal elements, repeated units at a single locus or scattered throughout the genome. The amplification of the gene for epidermal growth factor receptor (EGFR) is a common finding in glioblastomas and the amplified gene copies appears as double minutes. The aim of this study was to investigate the different patterns of EGFR amplification in $\mathbf{4 0}$ cases of glioblastoma using FISH analysis in metaphases and paraffin sections, and to investigate the relationship of gene copy number with gene expression profile. The analysis of copy number alterations of EGFR was validated by quantitative PCR and SNP microarrays. We observed that in $42 \%$ of the cases, the type of amplification of EGFR was as double minute chromosomes. In addition, we detected another type of amplification, with extra copies of EGFR inserted in different loci of chromosome 7 , present in $28 \%$ of cases. In this form of amplification, the number of copies is small, and the percentage of cells with EGFR amplification is rarely more than $15 \%$. This model of amplification could correspond to a variant of the insertion mechanism, or a consequence of a process of duplication. Our results suggest that this mechanism could represent an early stage of amplification in glioblastomas. Overall, we found a close correlation between EGFR gene copy-number alterations and the level of EGFR protein expression. However, all cases with a high level of mRNA exhibited strong expression for the EGFR protein, and most cases with a low level of mRNA showed no overexpression of EGFR protein. Modern Pathology (2010) 23, 856-865; doi:10.1038/modpathol.2010.62; published online 19 March 2010
\end{abstract}

Keywords: glioblastoma; EGFR amplification; insertion; microarrays; SNPs

Amplifications are mutations that result in multiple copies of genes in chromosomal regions (amplicons) and induce overexpression in cancer cells. The amount of overexpressed genes in amplified regions varies between different types of cancers, and gene expression, in general, is regulated significantly by DNA copy-number alterations., ${ }^{1,2}$ Chromosomal abnormalities associated with DNA amplification

Correspondence: Professor C Lopez-Gines, Department of Pathology, University of Valencia, Facultad de Medicina y Odontología, Avda. Blasco Ibañez 15, Valencia 46010, Spain.

E-mail: concha.lopez@uv.es

Received 2 November 2009; revised and accepted 28 January 2010; published online 19 March 2010 can be organized as extrachromosomal copies, called double minutes (dmins); in tandem arrays as head-to-tail or inverted repeats within a chromosome, often forming a cytologically visible, homogeneously staining region (HSR); or distributed at various locations in the genome (distributed insertions). ${ }^{1,3,4}$ The most frequently amplified genes in cancers are MYCN, ERBB2 and EGFR. ${ }^{4}$

The epidermal growth factor receptor (EGFR) is a cell membrane receptor with intrinsic protein tyrosine kinase activity, which has been the subject of rigorous investigation due to its involvement in several human cancers and its potential as a target of therapy. ${ }^{5}$ The activation of EGFR is induced by ligand binding that leads to tightly regulated 
stimulation of proliferative and survival cell signaling pathways; most notably the RAS/RAF/ MEK/MAPK and PI3K/Akt. ${ }^{6,7}$

Glioblastoma multiforme continues to be the most common primary brain tumor in adults with a rapidly progressive and fatal course despite current therapies. The molecular genetics of this tumor is under intense investigation. The EGFR gene (7p12.1) is frequently overexpressed, amplified and very rarely mutated in glioblastomas that arise de novo (primary glioblastoma). ${ }^{8}$

The first evidence of gene amplification in glioblastoma was provided by cytogenetic analyses that exhibited the presence of dmin. ${ }^{9,10}$ Molecular screening for gene amplification revealed frequent amplification of the EGFR, observed in about $35-70 \%$ of glioblastomas. ${ }^{8,11,12}$ Differences in the frequency of EGFR amplification are most probably due to different methods used, such as Southern blot, polymerase chain reaction (PCR), and fluorescent in situ hybridization (FISH).

The overexpresssion of EGFR protein is usually associated with gene amplification in glioblastomas and these two parameters have been studied as potential prognostic indicators. ${ }^{13,14}$ However, it is unclear whether EGFR overexpression would be an accurate prognostic factor in these tumors.

Fluorescent in situ hybridization is a powerful methodology to detect EGFR copy number abnormalities. This technique has been applied in glioblastomas, in touch preparation and paraffin sections, showing considerable heterogeneity in EGFR copy number, although most of the cases considered as amplified presented a high number of copies for this gene. ${ }^{6,11,14,15}$ In addition, FISH analysis using EGFR probe on tumor metaphases has been applied in a small number of glioblastomas, exhibiting the presence of signals on dmin, although HSRs have not been documented in metaphases of glioblastoma. ${ }^{15,16}$ Recent introduction of oligonucleotide microarrays designed for whole-genome genotyping of single nucleotide polymorphisms (SNPs) has facilitated the measurement of copy number changes at thousands of SNP loci. ${ }^{17,18}$

We performed FISH analysis using an EGFR probe during metaphases of primary cultured cells and in paraffin sections from 40 cases of primary glioblastomas. The aim of this study was to investigate differences in the pattern of EGFR amplification in this tumor. We also aimed to compare the EGFR copy number alterations, mRNA level and protein expression status, and to correlate the FISH data with the clinical and histopathological parameters in this subset of tumors.

\section{Materials and methods}

\section{Patient Population and Pathological Study}

Tumor samples were obtained from 40 patients with glioblastoma from the Clinic Hospital of Valencia.
All cases were newly diagnosed. None of the patients had received chemotherapy or radiotherapy before surgery. The study was reviewed and approved by Institutional Ethics Committee of the University of Valencia and Clinic Hospital of Valencia. The Karnofsky performance status (KPS) was calculated at the time of diagnosis. All patients underwent surgical removal of the tumor. Subsequently, radiotherapy with 50-65 Gy focal doses was applied to 36 patients. Chemotherapy, including BCNU (bis-chloroethylnitrosourea) or temozolomide, was administered in 31 patients. Survival times were calculated from the day of diagnosis of the glioblastoma to the end date of this study.

The tumor tissue was immediately fixed in neutral-buffered formalin, embedded in paraffin, sectioned and stained with hematoxylin and eosin. The samples were categorized according to the WHO classification ${ }^{19}$ and diagnosed as glioblastoma multiforme. Mitotic index values were obtained by counting the total number of mitotic figures in the tumor cells in 10 high-power fields (HPF) and in two different sections. The values are the mean number of mitoses per 10HPF.

The immunohistochemical study was performed on paraffin-embedded sections using the avidinbiotin peroxidase method. The study was carried out using antibodies against glial fibrillary acidic protein (GFAP; Dako, Glostrup, Denmark), Ki-67 (MIB-1, Dako), and monoclonal mouse anti-human EGFR (clone H11, Dako), which recognizes the wildtype EGFR and the deletion-mutant form of the receptor (EGFRvIII).

Proliferation index was evaluated using MIB-1 antibody staining and was calculated by determining the percentage of immunopositive nuclei. The expression of EGFR was quantified according to the intensity of staining and number of staining cells, as: 0 (no staining), 1 (light or focal), 2 (moderate), and 3 (strong). Scores of 0 or 1 were defined as no overexpression and scores of 2 and 3 as overexpression. ${ }^{13}$

\section{Fluorescence In Situ Hybridization}

To evaluate EGFR gene status, dual-color FISH analysis was performed on primary cultured cells and on paraffin sections from 40 samples. The FISH analysis was carried out using the LSR EGFR Spectrum Orange/CEP 7 Spectrum Green Probe from Vysis (Abbott Laboratories, Downers Grove, IL, USA; Cat. No. 32-191053).

Cultured cells were treated with Colcemid (0.02 $\mu \mathrm{g} / \mathrm{ml}$; Gibco BRL) for $80 \mathrm{~min}$ and chromosomes were prepared by conventional fixation. The paraffin embedded tissues were cut into 5- $\mu \mathrm{m}$ sections and these were mounted on Supoerfrost/ Plus microscope slides (Microm International). Hybridizations were performed according to the manufacturer's instructions. Counterstaining of 
nuclei was carried out using DAPI. The fluorescent signal was detected using a photomicroscope, Axioplan 2 and Axiophot 2 (Zeiss), equipped with a set of the appropriate filters. Signals were counted in 100-200 cultured cell nuclei, in all the possible metaphases and in 100 non-overlapping tumor cell nuclei in the paraffin sections. The mean signal number for the EGFR gene and CEP 7 was calculated for each case, followed by the calculation of EGFR gene/CEP 7 ratio. The EGFR gene was quantified as amplified in individual cells when the EGFR/ control signal ratio was greater than $2 .{ }^{14}$

\section{SNP Array Analysis for Assessment of EGFR Copy Number Status}

Nucleic acid isolation and microarray experiment Fresh frozen tumor tissues $(10-20 \mathrm{mg})$ from 20 glioblastoma patients were used to extract high molecular weight, genomic DNA using DNeasy kit extraction (Qiagen, Valencia, CA, USA). The quality of DNA was assessed by electrophoresis in $1 \%$ agarose gel. A total of $250 \mathrm{ng}$ DNA was used for hybridization on Genechip Human Mapping $100 \mathrm{~K}$ as recommended by the manufacturer (Affymetrix, Santa Clara, CA, USA). The mapping $100 \mathrm{~K}$ set provides a broad coverage of the human genome (92\%), with an average distance between SNPs of $23.6 \mathrm{~kb}$. This set includes two arrays, each with more than 50000 SNPs. The processing was performed following the manufacturer's instructions. Arrays were scanned using GeneChip Scanner 3000. GeneChip operating software was used to define Absent or Present Call and generate CEL files.

\section{Data analysis}

The CEL files were imported into dChipSNP array analysis software. ${ }^{20}$ Arrays were normalized against the array with median overall intensity (Baseline array) using the invariant set normalization method. ${ }^{21}$ Probe set signal intensity was obtained by using a model based on expression index (PM/MM) method. ${ }^{22}$ Average array call rate was $>95 \%$. A Hidden-Markov model was used to identify LOH regions from unpaired tumor samples, taking into account SNP intermarker distance, SNP heterozygosity rates, and the haplotype structure of the genome. ${ }^{23}$ We used $60 \mathrm{CEPH}$ parents as normal reference genotype and we removed inferred $\mathrm{LOH}$ regions consistent with $95 \%$ of homozygous markers in normal reference samples. Copy number alterations at each SNP locus were determined using the probe-level signal intensity data. ${ }^{24}$ Reference signal distribution was obtained using a trimmed analysis with $80 \%$ of the samples.

\section{Real-Time Quantitative PCR}

Tumor tissues from 25 patients with glioblastoma were frozen at $-80^{\circ} \mathrm{C}$. Tumor DNA was extracted from 10-15 mg of the tumor tissue using DNeasy kit extraction (Qiagen). Quantitative PCR (qPCR) was performed using an ABI Prism 7900 HT Fast RealTime PCR System (Applied Biosystem, Foster City, CA, USA) to analyze EGFR copy number. Primer sequences for EGFR were: forward: 5'-GTGCAG ATCGCAAAGGTAATCAG-3'; reverse: 5'-GCAGA CCGCATGTGAGGAT- ${ }^{\prime}$; and probe: $5^{\prime}$-FAM-CCC CTCCCCGTATCTC-MGB-3' ${ }^{\prime}$. Primer sequences for RNase $P$ and GAPDH, used as reference genes, were purchased as TaqMan RNase P and GAPDH Detection Reagents, respectively (P/N: 4316831).

Target and reference genes were amplified in separate wells. Each $20-\mu \mathrm{l}$ assay contained $20 \mathrm{ng}$ of genomic DNA, $900 \mathrm{nM}$ each of forward and reverse primers for the gene (RNase P, GAPDH as reference gene, and EGFR as target gene), and $250 \mathrm{nM}$ of labeled gene-specific probe in $1 \times$ TaqMan Universal PCR Master Mix. The TaqMan universal PCR Master Mix (Applied Biosystems) contained AmpliTaq Gold DNA polymerase, AmpErase uracyl- $N$ glycosylase, deoxynucleotide triphospates, with dUTPs that replace dTTPs, and optimized buffers.

Individual samples were run in triplicate. The PCR conditions were: $2 \mathrm{~min}$ at $50^{\circ} \mathrm{C}$ (initial incubation for activate AmpErase), $10 \mathrm{~min}$ at $95^{\circ} \mathrm{C}$ (activation of AmpliTaq Gold DNA polymerase) followed by 40 cycles of $15 \mathrm{~s}$ at $95^{\circ} \mathrm{C}$ (melt), and $1 \mathrm{~min}$ at $60^{\circ} \mathrm{C}$ (anneal/extend). Real-time data were collected using the SDS 2.1 software. Each replicate was normalized to reference gene (RNase $P$ and GAPDH) to obtain $\Delta C_{\mathrm{t}}$ value, and average $\Delta C_{\mathrm{t}}$ value for each sample (from the three replicates) was calculated. All samples were normalized to a calibrator sample to determine $\Delta \Delta C_{\mathrm{t}}$ value. Relative quantity is $2^{-\Delta \Delta C_{\mathrm{t}}}$ and copy number is $2 \times$ (relative quantity). ${ }^{25}$

Theoretically, a normal sample has two copies for each gene. Such an assumption is right in cell lines, but taking into consideration that human tumor tissue samples may be contaminated by normal cells, we used a copy-number range approach and considered: homozygous deletion (HD) 0-0.4; borderline HD-loss of heterozygosity $(\mathrm{LOH})>0.4-0.6$; $\mathrm{LOH}>0.6-1.4$; borderline $\mathrm{LOH}$-normal $>1.4-1.6$; normal $>1.6-2.4$; borderline amplification $>2.4-2.6$; and amplification $>2.6$.

\section{EGFR mRNA Expression}

\section{Nucleic acid isolation}

The RNA was extracted from 19 glioblastoma samples. Five pilocytic astrocytomas without EGFR amplification were used as reference. For total RNA extraction, 10-20 mg of frozen tumor samples were homogenized with Ultra Turrax and total RNA was isolated using mirVANA kit (Ambion, Austin, TX, USA) following the manufacturer's instructions. Purified RNA was quantified by UV absorbance at 260 and $280 \mathrm{~nm}$ and RNA quality was assessed using a spectrophotometer and Agilent 2100 Bioanalyzer 
(Agilent Technologies, Palo Alto, CA USA). Samples with $28 S / 18 S$ ratio of $\geq 1.1$ and no evidence of ribosomal peak degradation were included.

\section{Microarray experiments}

Total RNA (1-15 $\mu \mathrm{g})$ was used to generate doublestranded cDNA. cDNA synthesis and cRNA labeling were performed using the protocol for one-cycle cDNA synthesis. Biotin-labeled cRNA $(20 \mu \mathrm{g})$ was fragmented and hybridized overnight to Affymetrix HU133plus2.0 GeneChip. Protocols were performed as recommended by Affymetrix. Arrays were washed, stained with streptavidin phycoerythrin, and scanned to generate an image file. Scan quality was assessed by the inspection of visible microarray artifacts, grid placement, background intensity, and housekeeping gene expression. GeneChip operating software (GCOS) was used to define Absent or Present Call and generate CEL files. Arrays with $<30 \%$ 'Present' call for 47000 probe sets and signal $3^{\prime} / 5^{\prime}$ ratio of GADPH control $0.5 \leq$ ratio $\leq 4.5$ were omitted.

\section{Data analysis}

The CEL files were imported into dCHIP program. Arrays were normalized against the array with median overall intensity (baseline array) using the invariant set normalization method. ${ }^{21}$ Probe set signal intensity was obtained using a model based on expression index (PM/MM) method. ${ }^{22}$ The EGFR probe sets with Present Call and expression level $\geq 10$ in $\geq 45 \%$ samples were selected. Probe sets that passed established criteria were: 1565484_x_at, 201983_s_at, 201984_s_at, 211551_at, and $211607 \_\mathrm{x} \_$at.

\section{Statistical Analysis}

In this study, we used no parametric test for Ki67 levels, EGFR copy number, EGFR transcript expression, and percentage of cells found to be EGFR positive by FISH. First, the Kruskal-Wallis test was used to assess significant differences among established groups, and then the comparisons among the pairs of groups were assessed by means of Mann-Whitney $U$-test. We used the Bonferroni method to correct the $P$-values to avoid type-I error.

Pearson's coefficient was used to assess the significant correlation between: EGFR copy number measured by qPCR, EGFR transcript expression and percentage of EGFR-positive cells measured by FISH. The ssignificance of the differential protein expression was contrasted by comparing the EGFR amplification type using Fischer's exact test (one tailed). Survival curves for amplification types, KPS groups $(\leq 80$ and $>80)$, and age $(<55$ and $\geq 55$ years) were evaluated using the Kaplan-Meier method and were compared with the log-rank test. The statistical tests significance was determined at
$P$-value $\leq 0.05$. The analysis was performed using SPSS 15.0 software (SPSS, Chicago, IL, USA).

\section{Results}

\section{Clinical and Histopathological Data}

There were 24 male and 16 female patients, reflecting the male predominance among glioblastoma patients. Patient age ranged from 24 to 73 years, with a mean age of 55.5 years. The tumor locations were parietal in 9 patients, temporal in 16, frontal in 10 , occipital in 3 , and intraventricular in 2 patients. Preoperative functional status was evaluated according to the KPS scale. A total of 30 cases scored 80-100, whereas nine scored 70 and one scored 50 . Of the patients, $90 \%$ received radiotherapy and $77.5 \%$ received chemotherapy. Survival ranged from 3 to 24 months, with a mean survival period of 10.5 months. All patients, except four, were dead when this study was carried out.

Histologically, all tumors demonstrated features of glioblastoma with pleomorphic, astrocytic tumor cells, prominent microvascular proliferation, and necrosis. Five cases showed morphological features of small cell glioblastoma (cases 6, 7, 15, 17, and 24). In each case, the expression of GFAP was confirmed in neoplastic cells. The number of mitoses ranged between 0 and 20, with a mean value of 6 . The mean Ki-67 percentage for the tumors was $31.6 \%$; range: 5-80\%. The overexpression of EGFR (score 2,3) was observed in 20 tumor samples (50\%; Table 1).

\section{EGFR Gene and Chromosome 7 Copy Number Alterations}

A total of 40 glioblastomas were analyzed successfully by FISH. The FISH results are listed in Table 1. Cells with EGFR amplification were seen in $70 \%$ of the cases. The metaphases from cultured cells showed: amplification as dmin, in 15 cases (Figure 1a-c), extra copies of EGFR inserted in the p or/and q arms of chromosome 7, in 11 cases (Figure 1d-f). Furthermore, two cases showed both types of amplification (Figure 1g). A total of 12 cases of 40 glioblastomas showed no amplification (Figure 1i).

On basis of the EGFR status, the gene copy number, and the type of amplification, cases were categorized into three groups: (i) high-level EGFR gene amplification and dmin (cases 1-17), (ii) low-level EGFR gene amplification and insertions (cases 18-28), and (iii) no EGFR gene amplification (cases 19-40). The fraction of amplified cells in group (i) ranged from 1 to $80 \%$ in cultured cells (Figure 1a) and from 1 to $92 \%$ in cells of paraffin sections (Figure 1j). Only cases 16 and 17 contained less than $10 \%$ amplified cells in paraffin sections (Figure 1k). Cells with at least 25 EGFR signals were seen in all amplified cases of group (i). There were cells with more than 100 EGFR signals in nine cases, 
between 50-100 in three cases, and 25-50 signals in three cases. The fraction of amplified cells in group (ii) ranged from 4 to $23 \%$ in cultured cells and from 6 to $16 \%$ in cells of paraffin sections. These amplified cells showed between 6-25 EGFR signals (Figure $1 \mathrm{~h}$ and $\mathrm{l}$ ).
There was considerable heterogeneity in the centromere 7 copy number in these tumors. Amplified cases presented disomy in two, disomy/trisomy in three, trisomy in 12, trisomy/polysomy in nine, and only polysomy in two cases. In total, an excess of chromosome 7 copies was observed in $92 \%$ of the

Table 1 EGFR amplification status (FISH, qPCR), chromosome 7, mRNA level, and protein expression in 40 cases of glioblastomas

\begin{tabular}{|c|c|c|c|c|c|c|c|c|c|c|c|}
\hline \multirow[b]{2}{*}{ Case } & \multicolumn{4}{|c|}{ Cultured cells } & \multicolumn{3}{|c|}{ Paraffin section } & \multirow[b]{2}{*}{$q P C R$} & \multirow[b]{2}{*}{$m R N A$} & \multirow[b]{2}{*}{$\begin{array}{l}E G F R \\
(I H C)\end{array}$} & \multirow[b]{2}{*}{$M I B-1 \%$} \\
\hline & $\begin{array}{l}\text { Type of } \\
\text { amp }\end{array}$ & $\begin{array}{c}\text { EGFR } \\
\text { copies/cell }\end{array}$ & $\%$ Of cells & CEP 7 & $\begin{array}{l}\text { Type of } \\
\text { amp }\end{array}$ & $\%$ Of cells & CEP 7 & & & & \\
\hline 1 & dmin & $>100$ & 80 & dis & $\mathrm{dmin}$ & 90 & dis & NA & NA & 3 & 40 \\
\hline 2 & dmin & $>100$ & 74 & tri/poly & dmin & 92 & tri/poly & NA & NA & 3 & 10 \\
\hline 3 & dmin & $50-100$ & 21 & tri/poly & NA & NA & NA & NA & NA & 2 & 15 \\
\hline 4 & dmin & $>100$ & 57 & tri & dmin & 70 & tri & 168.3 & 1834 & 3 & 35 \\
\hline 5 & dmin & $>100$ & 20 & tri & dmin & 37 & tri & 55.1 & 1282 & 3 & 25 \\
\hline 6 & dmin & $25-50$ & 65 & tri & dmin & 73 & tri & 81.2 & 1133 & 3 & 75 \\
\hline 7 & dmin & $>100$ & 8 & tri/poly & dmin & 36 & tri/poly & 81.8 & 2141 & 3 & 50 \\
\hline 8 & dmin & $50-100$ & 6 & tri/poly & dmin & 80 & tri/poly & 68.1 & 2516 & 2 & 75 \\
\hline 9 & NA & NA & NA & NA & dmin & 82 & tri/poly & 77.3 & NA & 3 & 10 \\
\hline 10 & dmin & $>100$ & 11 & tri/poly & dmin & 91 & tri/poly & 208.2 & 3125 & 3 & 15 \\
\hline 11 & NA & NA & NA & NA & dmin & 51 & tri & 62.6 & 3192 & 3 & 20 \\
\hline 12 & dmin & $>100$ & 60 & tri & no & no & no & 97.6 & 2660 & 3 & 10 \\
\hline 13 & dmin & $25-50$ & 75 & tri/poly & dmin & 73 & tri/poly & 33.9 & 2063 & 3 & 50 \\
\hline 14 & dmin & $25-50$ & 51 & tri & $\mathrm{dmin}$ & 60 & tri & 17.8 & NA & 3 & 50 \\
\hline 15 & dmin/ins & $50 / 8-10$ & $80 / 4$ & tri & $\mathrm{dmin} / \mathrm{ins}$ & 21 & tri & 74.6 & 859 & 2 & 75 \\
\hline 16 & dmin/ins & $>100 / 12-18$ & $9 / 13$ & poly & dmin/ins & $3 / 1$ & poly & NA & NA & 1 & 80 \\
\hline 17 & dmin & $>100$ & $<1$ & tri & dmin & $<1$ & tri & 3.4 & 144 & 0 & 75 \\
\hline 18 & ins & $6-8$ & 15 & dis & NA & NA & NA & NA & NA & 0 & 10 \\
\hline 19 & ins & $14-16$ & 4 & poly & ins & 6 & poly & NA & NA & 1 & 50 \\
\hline 20 & ins & $8-12$ & 17 & tri & ins & 8 & tri & NA & NA & 1 & 15 \\
\hline 21 & ins & $6-8$ & 4 & tri & NA & NA & NA & NA & NA & 0 & 10 \\
\hline 22 & ins & $6-8$ & 4 & dis/tri & NA & NA & NA & NA & NA & 0 & 50 \\
\hline 23 & ins & $6-10$ & 23 & dis/tri & ins & 10 & dis/tri & 3.2 & 120 & 1 & 50 \\
\hline 24 & ins & $15-25$ & 9 & dis/tri & ins & 7 & dis/tri & 3.9 & 179 & 3 & 5 \\
\hline 25 & ins & $8-10$ & 5 & tri & ins & 11 & tri/poly & 3.0 & 325 & 0 & 20 \\
\hline 26 & ins & $10-18$ & 12 & tri/poly & ins & 13 & tri/poly & 6.1 & 141 & 2 & 30 \\
\hline 27 & ins & $8-16$ & 23 & tri/poly & ins & 16 & tri/poly & 6.5 & 534 & 2 & 10 \\
\hline 28 & ins & $8-10$ & 22 & tri & NA & NA & $\mathrm{NA}$ & 3.7 & 440 & 2 & 20 \\
\hline 29 & no amp & $3-6$ & - & tri & no amp & - & tri & 2.0 & NA & 0 & 50 \\
\hline 30 & no amp & $3-10$ & - & tri/poly & no amp & - & tri/poly & 2.0 & NA & 0 & 10 \\
\hline 31 & no amp & $3-10$ & - & tri/poly & no amp & - & tri & NA & NA & 1 & 15 \\
\hline 32 & no amp & $3-10$ & - & tri/poly & no amp & - & tri/poly & NA & NA & 1 & 75 \\
\hline 33 & no amp & $3-10$ & - & tri/poly & no amp & - & tri/poly & NA & NA & 0 & 10 \\
\hline 34 & no amp & $3-10$ & - & tri/poly & no amp & - & tri/poly & 2.1 & NA & 1 & 25 \\
\hline 35 & no amp & $3-6$ & - & $\operatorname{tri}$ & no amp & - & tri & 2.0 & NA & 0 & 10 \\
\hline 36 & NA & NA & NA & NA & no amp & - & tri & 2.6 & 458 & 0 & 5 \\
\hline 37 & no amp & $3-10$ & - & tri/poly & no amp & - & tri/poly & 2.3 & 119 & 0 & 25 \\
\hline 38 & no amp & $2-4$ & - & dis & no amp & - & dis & NA & NA & 1 & 10 \\
\hline 39 & no amp & $3-6$ & - & tri & no amp & - & tri & NA & NA & 2 & 30 \\
\hline 40 & no amp & $3-6$ & - & tri & no amp & - & tri & NA & NA & 1 & 20 \\
\hline
\end{tabular}

amp, amplification; CEP 7, centromere chromosome 7; dis, disomy; dmin, doubles minutes; IHC, immunohistochemistry; ins, insertion; tri, trisomy; poly, polysomy; NA, not available; - , no cells with amplification.

\footnotetext{
Figure 1 Two different patterns of EGFR amplification in glioblastomas. Chromosomal status of this amplification. Probe for EGFR and centromere 7 were labeled with red and green respectively. (a) High-level amplification of the EGFR in interphase nuclei of cultured cells. (b) Metaphase showing trisomy 7 and EGFR amplification as dmin. (c) Polysomy 7 in a metaphase spread with high number of dmin. (d and e) Low-level of amplication: metaphases spread with extra copies of EGFR inserted in p and q arms of chromosomes 7 ((d), trisomy 7 with two chromosomes 7 crossed). (f) Multiple extracopies of EGFR inserted and distributed in the three chromosomes 7 in a metaphase spread. (g) Different interphase nuclei of cultured cells showing high- and low-level (arrow) of amplification. (h) Low-level of amplication: interphase nuclei showing increased copy number of EGFR. (i) Non-amplified tumor cells of paraffin section. (j) Tumor cells showing high-level amplification of the EGFR in paraffin section. (k) Isolated amplified tumor cells in paraffin section. (l) Tumor cells in paraffin section with low-level amplification/copy number gains with other cells exhibiting a normal copy number of the EGFR gene.
} 

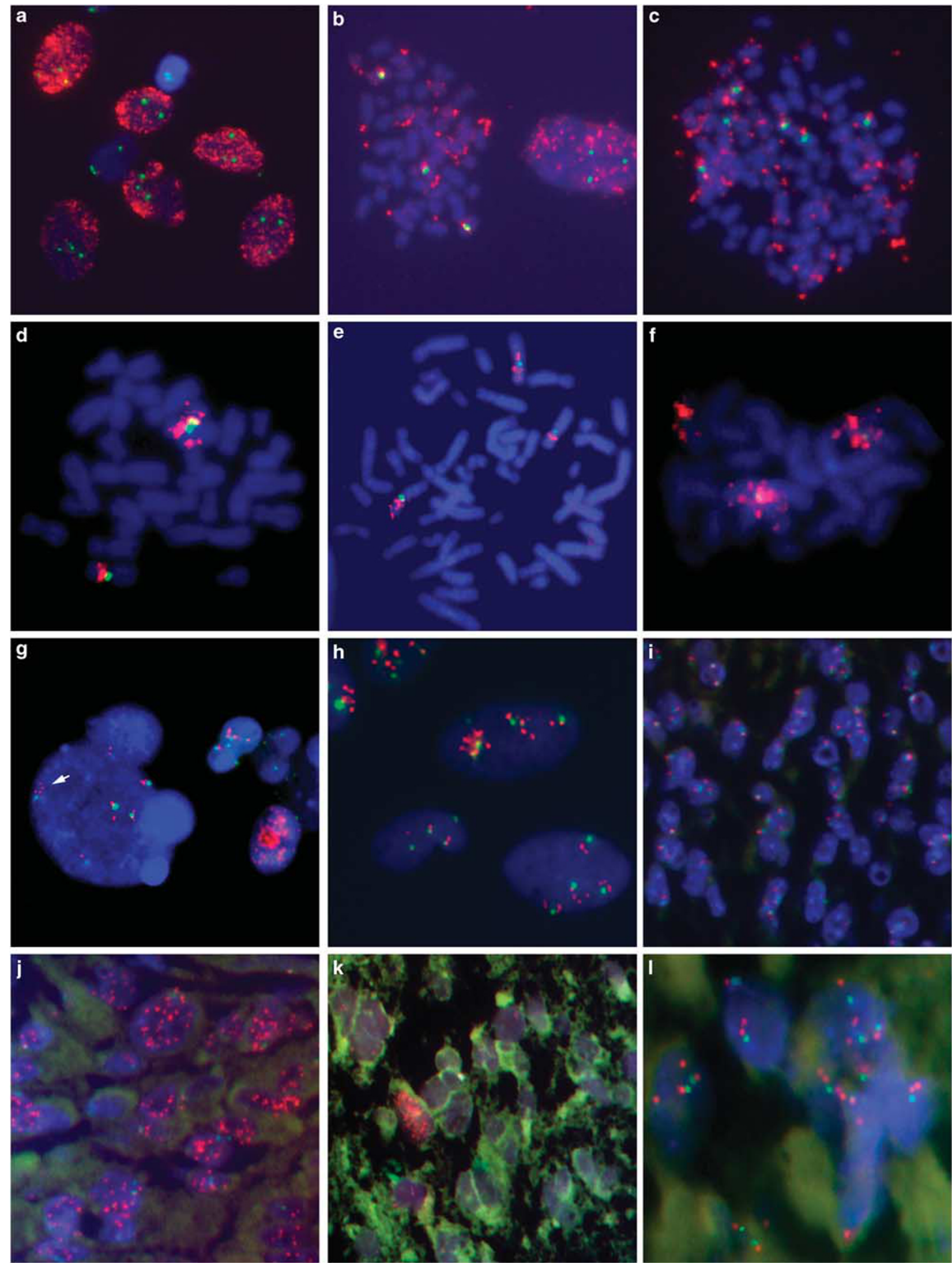

I 
amplified cases. Unamplified cases showed disomy in one, trisomy in four, and trisomy/polysomy in six cases. In this group, $90 \%$ cases presented an excess of chromosome 7 copies.

\section{Correlation of EGFR Gene Copy Number, EGFR mRNA Expression and EGFR Protein Expression}

Using SNP arrays, we inferred the presence of frequent copy-number gains in the region of the $E G F R$ locus. In tumor samples, an excess of EGFR gene copies was identified by copy number variation from the reference set and from the copy number of the control samples. An increase in the number of $E G F R$ copies was seen in tumors of group (i) (Figure 2A). Gene copy numbers at $7 \mathrm{p} 12.1$ were validated by qPCR. The qPCR results are listed in Table 1. Each case showed a strong correlation of these values with the copies demonstrated by FISH (Pearson's correlation coefficient $=0.77 ; \quad P$-value $\leq 0.01)$.

Gene expression data were available for 19 glioblastomas (Table 1). We found a significant, positive correlation between the EGFR copy number and the transcript gene expression (Pearson's correlation coefficient $=0.75 ; P$-value $\leq 0.01)$. The increases in EGFR dosage were associated with higher levels of EGFR transcript (Figure 2B). As expected, the higher percentage of cases of amplified EGFR detected by FISH was correlated with a higher gene expression (Pearson's correlation coefficient $=0.85 ; P$-value $\leq 0.01)$.

The EGFR protein expression was evaluated by immunohistochemistry. Cases with a high level of amplification, group (i), manifested EGFR overexpression, ie, scores $\geq 2$, except cases 16 and 17 . Cases with a low level of amplification, group (ii), had no EGFR overexpression in seven samples and EGFR overexpression in four samples. Cases that showed no amplification of EGFR, group (iii), did not express EGFR protein, except case 39 (Figure 2C). Overall, we found a close correlation between EGFR gene copy-number alterations and the level of EGFR protein expression in our collection of glioblastomas $(P$-value $=0.002$; Fisher's exact test $)$. However, all cases with a high level of mRNA exhibited strong expression for the EGFR protein, and most cases with a low level of mRNA showed no overexpression of EGFR protein.

a

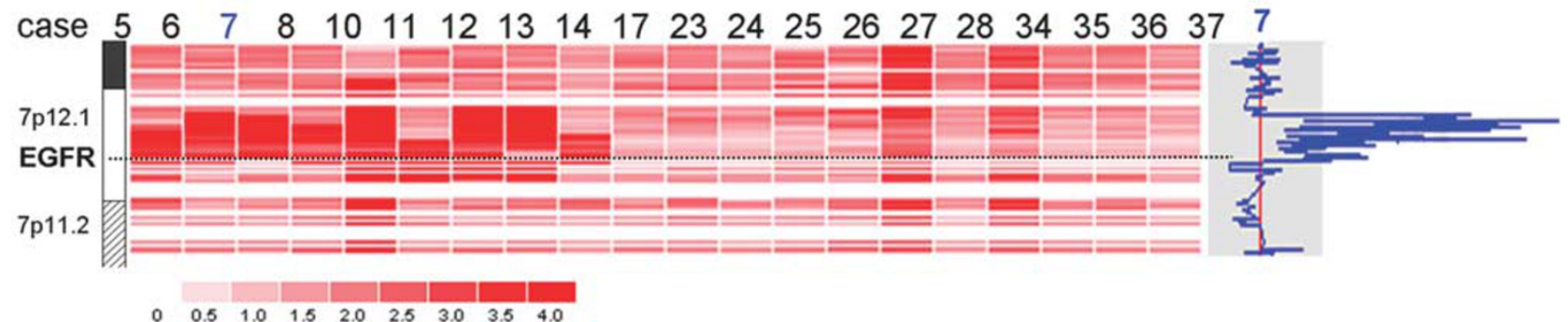

b

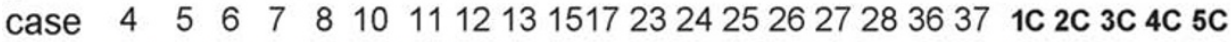
GBMGBMGBMGBMGBMGBMGBMGBMGBMGBMGBMGBMGBMGBMGBMGBMGBMGBMGBMAP AP AP AP AP

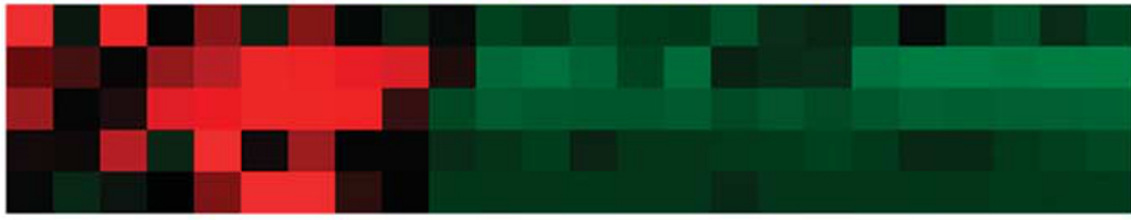

$1565484 \times$ x at, 201983 s_at, 201984_s_at, 211551_at, $211607 \times$ x at,

C
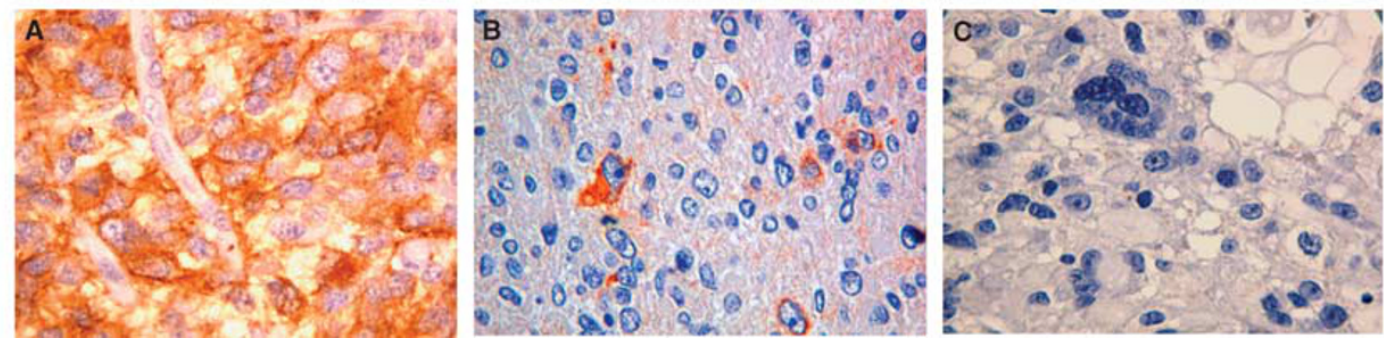

Figure 2 (a) Heat map integer copy number. Results of $100 \mathrm{~K}$ single nucleotide polymorphism (SNP) array analysis in 20 glioblastomas demonstrate genomic gains at 7p12.1, which includes the EGFR locus (cases 5-14), which correspond to the (i) group with high-level amplification. (b) EGFR gene expression heat map. Red bar denotes tumors with EGFR overexpression and green bar denotes glioblastomas with low expression of this gene. Probe sets are in rows, and samples in columns. (c) Immunoreactivity expression for EGFR: (A) Strong staining, (B) focal staining, and (C) no immunoexpression. Magnification: $\times 20$. 
Statistical Correlation of EGFR Gene Copy Alterations with Clinicopathological Parameters

The Kruskal-Wallis test was used to assess the differences among the three amplification groups and it showed significant differences in the percentage of cells with positive EGFR amplification detected by FISH, the EGFR copy number quantified by PCR, and the EGFR transcript expression assessed by microarrays ( $P$-value $\leq 0.01)$. In the percentage of EGFR-positive cells and EGFR copy number, all bilateral comparisons of the three amplification groups showed statistically significant differences, after the Bonferroni correction (i vs ii, i vs iii, ii vs iii; $P$-value $\leq 0.01$ ). The EGFR expression values of amplification types (i) and (ii) were statistically significant after the Bonferroni correction $(P$-value $\leq 0.01)$. No significant differences were found between types (ii) and (iii). The mean Ki-67 value of group (i) exhibited statistically significant differences with respect the rest $(P$-value $<0.05)$. Mean Ki-67 values for different groups were $41.8 \%$ for group (i), $24.5 \%$ for group (ii), and $23.9 \%$ for group (iii).

None of the survival curves showed any significant differences in established groups according to EGFR amplification type (i, ii, iii, i + ii $v s$ iii and i $v s$ $\mathrm{ii}+\mathrm{iii}), \mathrm{KPS}(\leq 80$ and $>80)$, and age $(<55$ and $\geq 55$ years). As expected, younger patients $(<55$ years) with KPS $>80$ showed a slightly higher survival rate. Interestingly, patients with EGFR amplification type (i) survived for the shortest duration; a mean of 8.7 months.

\section{Discussion}

Glioblastoma multiforme displays heterogeneous pathological features associated with complex molecular heterogeneity. Varying genetic alterations lead to the development of malignant phenotype expression and the amplification of EGFR is the most frequently involved oncogenetic pathway.

Amplified DNA can be organized as extrachromosomal elements (dmin), as concatenate homogenously staining chromosomal regions or scattered throughout the genome. ${ }^{1,3,4}$ According to the breakage-fusion-bridge model of amplification, the initiating event in HSR formation is double chromatid breakage at a fragile site or telomere erosion, ${ }^{2,26,27}$ fused sister chromatids and breaking of the anaphase bridges. ${ }^{28}$ Breakage-fusion-bridge cycles could then result in inverted amplified structures, ${ }^{29}$ and the mutated sister chromatids are distributed to the daughter cells giving rise to intra-tumor heterogeneity. ${ }^{30}$ Gene amplifications are also acquired by selection and unequal segregation of circular extrachromosomal chromatin (dmin and episomes). These elements are formed by looping out from the chromosomes. Dmin and episomes may also relocate in the genome after DNA double-stranded breaks to form HSR or distributed insertions. ${ }^{2,3,31}$ Instead of these above-mentioned mechanisms, N-MYC duplication located at 2 p24 has been described in neuroblastoma cell lines, as studied by FISH. ${ }^{32}$ Large direct duplications may arise by unequal sister chromatid exchange and seem to have a role during the initial stages in amplification. It is unclear whether duplication represents a prelude to amplification or an alternative pathway for activating the oncogenic potential of $M Y C .^{32,33}$ The single-copy N-MYC is retained at its original site on both, apparently normal, parental copies of chromosome 2 during amplification.

A characteristic cytogenetic feature of glioblastomas is the frequent presence of dmin, found in up to $50 \%$ of tumors. ${ }^{9,10}$ Molecular studies demonstrated the amplification of several genes in these tumors, especially of the EGFR gene; the amplified sequences were found located on dmin, analyzed in a small number of cases by in situ hybridization of tumor metaphases. ${ }^{15,16}$ However, studies by FISH in interphase nuclei are more frequent, displaying a considerable heterogeneity of EGFR copy number. $^{6,11,14,15}$ The cases presenting a diffuse rather than a clustered pattern of amplification within single cells support the idea that the most common pattern of EGFR amplification in glioblastoma biopsies is dmin. These dmins ranged in size from 0.7 to $2.1 \mathrm{Mb}$ and in level of amplification from 8- to 63-fold. ${ }^{15}$ The FISH signals differed from case to case, as intense and multiple, double, faint, and single. This suggests the complexity of dmin formation because both their size and the number of EGFR copies they contain, vary. Cell-to-cell variations are also likely to exist for a given tumor. ${ }^{11}$

To the best of our knowledge, this is the first study to analyze the status of the EGFR locus at chromosomal level by FISH in a series of 40 newly diagnosed glioblastomas. We observed that in $42 \%$ of the cases, the type of amplification is dmin with a variable number of copies affecting over $10 \%$ of the cells in most cases. Together with this type of amplification, we have demonstrated another type of amplification present in $28 \%$ of the cases, in which extra copies of EGFR inserted in different locus of chromosome 7 , both in $\mathrm{p}$ and $\mathrm{q}$ arms, were observed. In this form of amplification, the number of copies is small, and the percentage of cells with the amplification of EGFR rarely affects more than $15 \%$ of the cells. This model of amplification could correspond to a variant of the distributed insertion mechanism, or a consequence of a process of duplication similar to the one demonstrated in neuroblastoma cell lines. ${ }^{32}$ On the basis of these results, we suggest that this mechanism could be considered as an early stage of amplification in glioblastomas. This hypothesis is corroborated by the findings on both status of amplification, dmin, and extra copies, located in chromosome 7, in two cases. In glioblastomas, the cut-off points that define amplification are controversial, ${ }^{14,34}$ although the most commonly accepted criterion is that EGFR 
amplification exists when the EGFR gene/chromosome ratio per cell was $\geq 2$, or $\geq 15$ copies of $E G F R$ are present per cell in $\geq 10 \%$ of analyzed cells. ${ }^{35-37}$

Microarrays designed for the analysis of SNPs can also be applied in the detection of DNA copynumber alterations. Recent studies have been carried out on glioblastomas and different percentages of EGFR amplification have been detected.7,17,18,38 Our results confirm an excess of EGFR copies in cases with a high-level of amplification. The SNP loci with copy-number changes were validated by qPCR. Our cases presented high values of EGFR copies, as analyzed by qPCR, in cases with dmin, and low values in cases with insertions. We can confirm a close correlation between these values and those obtained by FISH. However, techniques such as array analysis of SNPs and dPCR (differential) are less likely to detect tumors with isolated EGFR-amplified cells or tumors with low-level amplification present in a small subpopulation of tumor cells. ${ }^{6}$

The integration of SNP data with gene expression microarray data is also required to understand the effects of gene regulation and transcription in amplification manifestation. The EGFR gene has been found to be expressed at a significantly higher level in glioblastomas. ${ }^{8,39}$ The increase in mRNA level caused by amplification is not always proportional to the number of gene copies; ${ }^{33}$ and the effects of the gene copy number on expression levels were more relevant for high-level amplifications in a gene-by-gene analysis; results that were also found in breast and prostate cancers. ${ }^{40,41}$ In our series, only the cases with dmin amplification, present in more than $10 \%$ of the cells, showed an increase of RNA expression.

The EGFR protein overexpression is usually associated with gene amplification in glioblastomas. ${ }^{6,14}$ In this study, all cases with EGFR amplification (group i), except two, showed EGFR overexpression; these two cases without EGFR overexpression presented less than $10 \%$ of amplified cells. In group (ii), only one-third of the cases showed EGFR overexpression; and in group (iii), only one case showed EGFR overexpression.

In addition, the relationship between trisomy/ polysomy of chromosome 7 and EGFR gene amplification has been analyzed. In our study, both amplified and non-amplified tumors showed trisomy/polysomy of chromosome 7 . This fact suggests that this alteration could be an initial event in the tumorigenesis of glioblastoma.

Amplification in general and amplicons in particular are important for both prognosis and targeted therapies. Specific amplicons, such as $M Y C N$ in neuroblastomas and $M Y C$ and ERBB2 in breast cancer, also have prognostic significance. ${ }^{4}$ The EGFR amplification status has been studied as a potential prognostic indicator. The majority of the early reports showed that amplification of the EGFR was associated with a poorer prognosis than that associated with non-amplified glioblastomas. Other studies failed to confirm this relationship. ${ }^{13,14,34,42}$ Our study did not show that the type of EGFR amplification led to significant differences in survival rates; although, interestingly, patients with EGFR amplification type (i) survived for the shortest time.

We propose that EGFR amplification is an important and frequent pathway in glioblastomas. This amplification is expressed as dmin in a subset of glioblastoma; however, we have observed another type of amplification of the EGFR gene. This is seen as distributed insertions of this gene in chromosome 7 . Detection of this pattern of amplification of EGFR may further improve the prognostic value of genomic diagnosis of this disease.

As amplification is often associated with poor prognosis and is a mechanism of resistance to therapies, it will be important to identify the genes or pathways (or both) that promote amplification in tumors, so that they might be targeted as part of a combination therapy to prevent the evolution of resistance to drugs designed to arrest or eradicate the tumor.

To evaluate the significance of this genomic aberration, it will be necessary to survey a large series of these primary tumors.

\section{Acknowledgements}

We thank Ms Ana Clari for her technical assistance. This study was supported by a grant from FIS, PI061134 (2006-2009), and eTUMOR:FP6-2002LIFESCIHEALTH 503094.

\section{Disclosure/conflict of interest}

The authors declare no conflict of interest.

\section{References}

1 Schwab M. Amplification of oncogenes in human cancer cells. Bioessays 1998;20:99-102.

2 Myllykangas S, Knuutila S. Manifestation, mechanism and mysteries of gene amplifications. Cancer Lett 2006;232:79-89.

3 Kuwahara Y, Tanabe C, Ikeuchi T, et al. Alternative mechanisms of gene amplification in human cancers. Genes Chromosomes Cancer 2004;41:125-132.

4 Albertson DG. Gene amplification in cancer. Trends Genet 2006;22:447-455.

5 Hynes NE, Lane HA. ERBB receptors and cancer: the complexity of targeted inhibitors. Nat Rev Cancer 2005;5:341-354.

6 Mizoguchi M, Betensky RA, Batchelor TT, et al. Activation of STAT3, MAPK, and AKT in malignant astrocytic gliomas: correlation with EGFR status, tumor grade, and survival. J Neuropathol Exp Neurol 2006;65:1181-1188.

7 TCGA (The Cancer Genome Atlas Research Network). Comprehensive genomic characterization defines human glioblastoma genes and core pathways. Nature 2008;455:1061-1068. 
8 Ohgaki H, Kleihues P. Genetic pathways to primary and secondary glioblastoma. Am J Pathol 2007;170: 1445-1453.

9 Bigner SH, Vogelstein B. Cytogenetics and molecular genetics of human brain tumors. Brain Pathol 1990;1:12-18.

10 Thiel G, Losanowa T, Kintzel D, et al. Karyotypes in 90 human gliomas. Cancer Genet Cytogenet 1992; 58:109-120.

11 Sauter G, Maeda T, Waldman FM. Short communication: Patterns of epidermal growth factor receptor amplification in malignant gliomas. Am J Pathol 1996;148:1047-1053.

12 Okada Y, Hurwitz EE, Esposito JM, et al. Selection pressures of TP53 mutation and microenvironmental location influence epidermal growth factor receptor gene amplification in human glioblastomas. Cancer Res 2003;63:413-416.

13 Shinojima N, Tada K, Shiraishi S, et al. Prognostic value of epidermal growth factor receptor in patients with glioblastoma multiforme. Cancer Res 2003;63: 6962-6970.

14 Layfield LJ, Willmore C, Tripp S, et al. Epidermal growth factor receptor gene amplification and protein expression in glioblastoma multiforme. Appl Immunohistochem Mol Morphol 2006;14:91-96.

15 Vogt N, Lefèvre SH, Apiou F, et al. Molecular structure of double-minute chromosomes bearing amplified copies of the epidermal growth factor receptor gene in gliomas. Proc Natl Acad Sci USA 2004; 101:11368-11373.

16 Muleris M, Almeida A, Dutrillaux AM, et al. Oncogene amplification in human gliomas: a molecular cytogenetic analysis. Oncogene 1994;9:2717-2722.

17 Wong KK, Tsang YT, Chang YM, et al. Genome-wide allelic imbalance analysis of pediatric gliomas by single nucleotide polymorphic allele array. Cancer Res 2006;66:11172-11178.

18 Wrensch M, McMillan A, Wiencke J, et al. Nonsynonymous coding single-nucleotide polymorphisms spanning the genome in relation to glioblastoma survival and age at diagnosis. Clin Cancer Res 2007;13:197-205.

19 Louis D, Ohgaki H, Wiestler O, et al. WHO Classification of Tumors of the Nervous System, 4th edn. IARC: Lyon, 2007.

20 Lin M, Wei LJ, Sellers WR, et al. dChipPSNP: Significance curve and clustering of SNP-array-based loss-of-heterozigosity data. Bioinformatics 2004;20: 1233-1240.

21 Li C, Wong WH. Model based analysis of oligonucleotide arrays: Expression index computation and outlier detection. Proc Natl Acad Sci USA 2001a;98:31-36.

$22 \mathrm{Li} \mathrm{C,} \mathrm{Wong} \mathrm{WH.} \mathrm{Model} \mathrm{based} \mathrm{analysis} \mathrm{of} \mathrm{oligo-}$ nucleotide arrays: model validation, design issues and standard error application. Genome Biol 2001b;2:8-14.

23 Beroukhim R, Ling M, Park Y, et al. Inferreing LOH from unpaired tumours using High Density Oligonucleotide SNP arrays. PLoS Comput Biol 2006;2:5.

24 Zhao X, Li C, Paez G, et al. An integrated view of copy number and allelic alterations in the cancer genome using single nucleotide polymorphism arrays. Cancer Res 2004;64:3060-3071.

25 Livak KJ, Schmittgen TD. Analysis of relative gene expression data using real-time quantitative PCR and the $2^{-\Delta \Delta C t}$ method. Methods 2001;25:402-408.

26 Coquelle A, Pipiras E, Toledo F, et al. Expression of fragile sites triggers intrachromosomal mammalian gene amplification and sets boundaries to early amplicons. Cell 1997;89:215-225.

27 Murnane JP, Sabatier L. Chromosome rearrangements resulting from telomere dysfunction and their role in cancer. Bioessays 2004;26:1164-1174.

28 Shimizu N, Shingaki K, Kaneko-Sasaguri Y, et al. When, where and how the bridge breaks: anaphase bridge breakage plays a crucial role in gene amplification and HSR generations. Exp Cell Res 2005;302: 233-243.

29 Toledo F, Le Roscouet D, Buttin G, et al. Co-amplified markers alternate in megabase long chromosomal inverted repeats and cluster independently in interphase nuclei at early steps of mammalian gene amplification. Eur Mol Biol Org J 1992;11:2665-2673.

30 Gisselsson D, Pettersson L, Hoglund M, et al. Chromosomal breakage-fusion-bridge events cause genetic intratumor heterogeneity. Proc Natl Acad Sci USA 2000;97:5357-5362.

31 Schwab M. Oncogene amplification in solid tumors. Cancer Biol 1999;9:319-325.

32 Corvi R, Savelyeva L, Schwab M. Duplication of NMYC at its resident site 2p24 may be a mechanism of activation alternative to amplification in human neuroblastoma cells. Cancer Res 1995;55:3471-3474.

33 Schwab M. MYCN in neuronal tumours. Cancer Lett 2004;204:179-187.

34 Smith JS, Tachibana I, Pohl U, et al. PTEN mutation, EGFR amplification, and outcome in patients with anaplastic astrocytoma and glioblastoma multiforme. J Natl Cancer Inst 2001;93:1246-1256.

35 Cappuzzo F, Hirsch FR, Rossi E. Epidermal growth factor receptor gene and protein and gefitinib sensitivity in non-small-cell lung cancer. J Natl Cancer Inst 2005;97:643-655.

36 Hirsch FR, Varella-García M, McCoy J, et al. Increased epidermal growth factor receptor gene copy number detected by fluorescence in situ hybridization associates with increased sensitivity to gefitinib in patients with bronchioloalveolar carcinoma subtypes: a Southwest Oncology Group Study. J Clin Oncol 2005;23:6838-6845.

37 Nagasaka T, Gunji M, Hosokai N, et al. FISH 1p/19q deletion/imbalance for molecular subclassification of glioblastoma. Brain Tumor Pathol 2007;24:1-5.

38 Kotliarov Y, Steed ME, Christopher N, et al. Highresolution global genomic survey of 178 gliomas reveals novel regions of copy number alteration and allelic imbalances. Cancer Res 2006;6619:9428-9436. Erratum in: Cancer Res 2007; 67:2389.

39 Pedersen MW, Pedersen N, Damstrup L, et al. Analysis of the epidermal growth factor receptor specific transcriptome: effect of receptor expression level and an activating mutation. J Cell Biochem 2005; 96:412-427.

40 Hyman E, Kauraniemi P, Hautaniemi S, et al. Impact of DNA amplification on gene expression patterns in breast cancer. Cancer Res 2002;62:6240-6245.

41 Wolf M, Mousses S, Hautaniemi S, et al. Highresolution analysis of gene copy number alterations in human prostate cancer using CGH on cDNA microarrays: impact of copy number on gene expression. Neoplasia 2004;6:240-247.

42 Simmons ML, Lamborn KR, Takahashi M, et al. Analysis of complex relationships between age, p53, epidermal growth factor receptor, and survival in glioblastoma patients. Cancer Res 2001;61:1122-1128. 